\title{
ACUTE PSYCHOSES IN ELDERLY PATIENTS WITH ALCOHOL WITHDRAWAL SYNDROME
}

Valeriy N. Kuzminov (Ukraine) Ph.D., Senior Researcher, Department of Emergency Psychiatry and Narcology, SI "Institute of Neurology of Psychiatry and Narcology, National Academy of Medical Sciences of Ukraine"

The psychoses in patients with dependence of alcohol are in many cases polyethiologic, this is especially common in the elderly age due to the accumulation of various somato-neurological pathologies. In elderly patients, notably after a retirement, dependency is formed more quickly, and the existing one is more severe due to the breakdown of the dynamic stereotype of life and a decrease in the number of inhibiting factors in alcohol consumption. The severity of the course of such psychoses, regardless of the etiology, manifested by delirious syndrome, is well known. Estimation of the leading factor in the emergence of acute psychosis in patients with alcohol dependence in the state of alcohol withdrawal is of great practical importance for therapeutic issues.

Aim of study. The study presents an attempt of solution of the problem of acute psychotic disorders in elderly patients with alcohol dependence, by studying their structure, pathogenetic factors and developing on these basis optimal algorithms for their diagnosis, treatment and prevention of relapse.

Materials and methods. Under supervision were $80 \mathrm{men}$, dependent on alcohol, in the state of withdrawal with acute psychotic disorder the average age - $(66,8 \pm 5,7)$ years, the average age of alcohol abuse - $(21,4 \pm 5,2)$ years. Research methods: clinical, psychopathological, laboratory, neuroimaging (to exclude acute incranial pathology). Medical care for patients was provided in intensive care wards of the Center for Emergency Psychiatry of Kharkiv Regional Hospital No.3.

Results. In the process of clinical and statistical analysis of data on the urgent hospitalization of patients with alcohol dependence on acute psychotic disorder, it has been established that a significant part of these disorders ( $25 \%$ of cases) is due to reasons not directly related to the use of alcohol, while the contribution made by these patients with non-alcoholic psychotic disorders to mortality is significantly $(p<0.001)$ higher than in patient with alcohol withdrawal delirium. During the course of the study, criteria for differential diagnosis, principles of therapy and prevention of relapses of acute psychotic disorders in patients with alcohol dependence in the withdrawal were developed on the basis of studying the factors influencing their formation and development of mechanisms of their formation, as well as clinical-psychopathological, pathopsychological , electrophysiological, laboratory data. It was established that with probability $\mathrm{p}<0.05$ of the groups studied with metallic psychoses and psychoses that were caused not by alcohol use were: focal symptoms diagnostic coefficient (DC) - 6,5, meningeal signs DC - 5,4, stunning DC - 6,5, independence somatoneurologic symptoms of consumption before DC - 4,0. The clinical-psychopathological structure of acute psychotic disorders in patients with alcohol dependence in the state of alcohol withdrawal was studied.

A comparison of clinical and dynamic patterns of alcohol dependence and psychopathological disorders in the structure of alcohol psychosis were studied. When comparing patients in whom alcohol dependence developed after retirement, it was found that they had a more pronounced psychopathological picture and a relatively favorable course compared with patients with a long history of alcohol abuse. The influence of the factors of permanent and temporary on the clinical picture and the pathology of alcoholic delirium have been studied. At the same time, the severity of the clinical picture depended heavily on the severity of alcohol dependence on alcohol according to the scales, so the overall severity of the dependence syndrome among patients with the state of withdrawal of alcohol with abortive delirium was $(33,94 \pm 1,21)$ points against $(38,17 \pm 1,31$ ) scores among patients with a classic alcohol state (differences are statistically significant $p<0.05$ ). It was found that in the severe state of withdrawal of alcohol with delirium type GAE (F 10.43 according to ICD-10) is significantly more $p<0.01$ than in other stages of the withdrawal of alcohol with delirium (F 10.4 in accordance with ICD-10) there are symptoms that are the criteria for differential diagnosis of metalcoholic psychoses of patients with acute psychotic state with cerebral pathology (F05 according to ICD-10). Due to the fact that the state of alcohol withdrawal played a significant role, at least it was a provoking factor in most cases, the diagnosis was made F058 according to ICD-10/ At studying the course of severe conditions for the abolition of alcohol with delirium type acute encephalopathy (GAE), it was found that reliable ( $p<0,0001)$ marker predictors GAE in patients in the state of withdrawal of alcohol differ among themselves in the frame of time, since they allow predict the formation this pathological condition. The influence of the transferred psychotic disorders on the course of alcohol dependence is studied. In the early period after a psychotic disorder, the attraction to alcohol had less severity mainly due to the behavioral and ideatonic components (the differences were statistically significant $\mathrm{p}<0.05$ for the Cherednichenko-Altshuller glossary). The number of repeated psychoses was higher in patients undergoing the first lung (abortive delirium). In patients who suffered the first severe delirium, further in the course of the disease, the number of repeated psychoses significantly decreased. At the same time, the factor that increased the progressiveness of alcohol dependence was persistent cognitive impairment in this group (according to MMSE), fewer spontaneous remissions (differences are statistically significant $p<0.01$ ), greater accumulation of chronic somato-neurological pathology associated with alcohol abuse.

The influence of the transferred psychotic disorders on the development of stable cognitive impairments has been studied. It was found that the prognostic features of the development of a psycho-organic syndrome after an acute psychotic disorder are a no psychotic disturbance of consciousness in the structure of psychosis, long-term psychomotor agitation, amentive syndrome, and neurological disorders: focal neurological symptoms, nystagmus, anisocoria, strabism, meningeal signs. The algorithm of providing the principles of therapy and prevention of the development of acute psychotic disorders in patients with alcohol dependence is developed. It is shown that the effectiveness of the use of antipsychotics in patients with psychotic disorders in patients with alcohol dependence (F05, F10.5 according to ICD-10) is more effective than in patients with a state of withdrawal of alcohol with delirium where benziodesepines and drugs for anesthesia (sodium thiopental, sodium oxibutyrate) were more effective. A combination of sedatives is preferable to increasing the dose of one drug. The development of acute psycho-organic syndrome was determined by the rate of termination of psychomotor excitation (correlation coefficient - 0.86) and the total dose of sedative drugs used for treatment (correlation coefficient +0.75$)$. Therefore, a differentiated estimation of sedation level in patients with metalcoholic psychosis with the help of RASS and CAM-ICU scales for optimization of sedative therapy were provided. It was found that patients with signs of developing acute alcoholic encephalopathy (F10.43 according to ICD-10) needed more sedation $-4,-5$ on the RASS scale than patients with classical alcohol withdrawal status with delirium and (F10.4 according to ICD-10) recommended level of sedation $-2,-3$, on the scale of RASS and $0,-1$ for acute psychotic.

The effectiveness of craniocerebral hypothermia in the treatment of severe withdrawal of alcohol with delirium type GAE was confirmed during investigation. The occurrence or increase of cognitive deficit after acute psychosis in the state of alcohol withdrawal primarily depended on the total duration of the psychotic state, the presence of cardiovascular insufficiency (manifested by a significant decrease in blood pressure).

\section{Conclusions}

1. With the general similarities of the clinical picture of delirium connected with somato-neurological diseases and states of alcohol withdrawal with delirium in elderly patients, careful study of the psychopathological structure and patterns of development of psychosis allow to differentiate these disorders.

2. The criteria that allow differentiation of delirium connected with somato-neurological diseases and the state of alcohol withdrawal with delirium were less severe vegetative signs of the state of withdrawal, correlation of the duration of psychosis with somatoneurological pathology, the presence of amentive syndrome from the onset of psychosis, the peculiar properties and dynamic of psychomotor excitement and particularity of psychotic symptoms.

3. All prolonged acute psychotic states in patients with alcohol dependence, which arise in the state of alcohol withdrawal, have common features and are a consequence of decompensation of somatoneurological pathology.

4. The differentiated approach to treatment with acute psychosis in elderly patients with alcohol withdrawal syndrome and reduce treatment time and prevent the growth of cognitive deficiency. 